\title{
"Can you hear me now?" Video conference coping strategies and experience during COVID-19 and beyond
}

\author{
Hannah Johns ${ }^{\mathrm{a}, \mathrm{b}, 1}$, Emma L. Burrows ${ }^{\mathrm{b}, 2}$, Venesha Rethnam ${ }^{\mathrm{a}, \mathrm{b}, 3}$, \\ Sharon Kramer ${ }^{\mathrm{a}, \mathrm{b}, \mathrm{c}, 4}$ and Julie Bernhardt ${ }^{\mathrm{a}, \mathrm{b}, *, 5}$ \\ ${ }^{a}$ National Health and Medical Research Council (NHMRC) Centre of Research Excellence in Stroke \\ Rehabilitation and Brain Recovery, Melbourne, Australia \\ ${ }^{\mathrm{b}}$ Florey Institute of Neuroscience and Mental Health, Parkville and Austin Campuses, Melbourne, Australia \\ ${ }^{c}$ School of Nursing and Midwifery, Deakin University, Melbourne, Australia
}

Received 22 February 2021

Accepted 26 July 2021

\begin{abstract}
.
BACKGROUND: During COVID-19 the workforce quickly adapted to using existing video-conferencing tools in order to work from home.

OBJECTIVE: To explore use and experience of remote video-facilitated work practices in response to COVID-19: termed 'COVID Response Zoom-style Interactions' (CRAZI).

METHODS: A cross sectional study via a 66-item online survey for health and medical research and education sector workers. The survey included 8 sections: 1) pre-COVID video-conferencing meeting habits, 2) CRAZI meeting habits, 3) socialising, 4) CRAZI fashion, 5) behaviour standards, 6) family life, 7) future work, 8) participant demographics. Main outcomes were pre-COVID to CRAZI differences in frequency, length and type of video-based meetings, and video-conferencing experience. RESULTS: 202 participants, mostly Australian (median age 36-45, IQR 26-55 years) completed the survey. Women-to-men ratio was $3: 1,44.6 \%$ had children. COVID-19 changed video-conferencing frequency and maximum meeting size. Most participants found CRAZI meetings tiring and hard. Casual clothes dominated dress code (71.1\%), pets were commonly seen. "Can you hear me now" was a commonly heard phrase. Good and bad behaviour were described, with formal codes of CRAZI conduct missing (58.7\%) or unknown (21.9\%). 76.6\% of participants observed a child interrupting a CRAZI meeting, parents were mostly female. Despite challenges, most participants $(76.6 \%)$ favoured video-conferencing post-pandemic, but preference for continuing to work from home varied.

CONCLUSIONS: CRAZI work, while tiring, has fostered different work-practices that may continue beyond the pandemic. Working from home with children adds joy for others, but complexity for workers. Pets may help owners and co-workers cope with the pandemic.
\end{abstract}

Keywords: Zoom fatigue, video conferencing behaviour, work-from-home

\footnotetext{
${ }^{1}$ ORCID: 0000-0003-2135-0504.

${ }^{2}$ ORCID: 0000-0002-6675-4679.

${ }^{3}$ ORCID: 0000-0002-0493-8536.

${ }^{4}$ ORCID: 0000-0003-2795-6259.

${ }^{5}$ ORCID: 0000-0002-2787-8484
}

*Address for correspondence: Julie Bernhardt, Florey Institute of Neuroscience and Mental Health, Austin Campus, 245 Burgundy Street, Heidelberg, Victoria, 3084, Australia. E-mail: Julie.bernhardt@ florey.edu.au. 


\section{Introduction}

COVID-19 has changed the way we work. In Australia, the first lockdown saw a government mandated work from home policy, "If you can work from home, stay at home". Before COVID-19 we has already begun to see a trend towards flexible work arrangements including working from home. There has been a steady increase in people working from home in the UK [1], Europe [2] and United States [2, 3] with reports ranging from 20-30 percent of people working from home or teleworking from a location other than the employer's premises [2]. The increase in remote working arrangements has been made possible by technical advancements [4]. Additionally, a higher demand for more flexible work arrangement by employees also underpins the shift to higher rates of employees working from home. A large survey carried out in multiple high-income countries showed that $84 \%$ of respondents think that flexible work arrangements should be the norm [5].

Several positive aspects to flexible working arrangements have been reported. For example, working from home is associated with greater job satisfaction and people with flexible working arrangements report higher rates of enthusiasm for the job [6]. It has also been suggested that remote working leads to an improvement in creativity and productivity [7]. On the other hand, working from home also has its challenges. In a survey of 43 remote workers, respondents indicated that the challenges they encountered when working remotely were related to collaborating and communication with others, making the technology work, finding information, and balancing work and life [8]. In a qualitative study by Grant et al. [9], eleven remote workers across different organisations and industries were interviewed about the psychological impacts of working remotely. Thematic analyses showed that adverse impacts were related to overworking and limited time to recover [9].

At the start of the COVID-19 pandemic people did not have a choice but were instead forced to work from home. Additionally, schools were closed, and children needed to be home schooled. The workforce scrambled to create home workstations and get back to work in a new and sometimes chaotic work environment. Face-to-face interactions, the mainstay of our social lives and work practices, were gone and existing video-conferencing tools were rapidly adapted to become the backbone communication tool. As we experienced these rapid changes, the opportunity to capture both the funny and challenging aspects of pivoting to video-based work from home was an important area to explore.

\section{Objective}

This survey explores the experience of remote video-facilitated work (and social) practices during the early COVID-19 work from home period; called 'COVID response and Zoom-style interactions' (CRAZI). We aimed to compare work practices pre-CRAZI, with CRAZI work and social practices, document CRAZI standards of behaviour and dress, explore the impact of parenting and home schooling on work, and interest in continuing to work from home after the pandemic.

\section{Method}

The 66-item survey tool (see Supplementary Materials for tool) developed in Google forms included mostly multiple choice questions. Additional short answer, open fields for CRAZI fashion and behaviour allowed for examples. Items could be skipped should participants wish.

This project was retrospectively approved by The University of Melbourne, Human Research Ethics Committee, Ethics ID 2057602.1.

With an uncertain response rate given the timing at the start of the pandemic, we had no formal sample size estimates. Participant selection was based on a convenience sample. The survey link was distributed via email, internal advertising and social media to our peers. Recruitment period was 4.5 months.

Summary statistics report quantitative data. New and experienced users were separated for pre-CRAZI to CRAZI video-conferencing experience comparisons. $\mathrm{R}$ Version 4.0.1 was used for all statistical analyses [10].

Open field data were coded and analysed using a thematic approach by an experienced researcher (JB) who developed a coding frame and interpreted key themes.

\section{Results}

The survey opened on May 10, 2020 and closed August 4, 2020. Two hundred and four responses were recorded, 2 of these were duplicates and were removed, leaving 202 responses. Most respondents completed all items. Free text questions 
Table 1

Summary demographics of participants

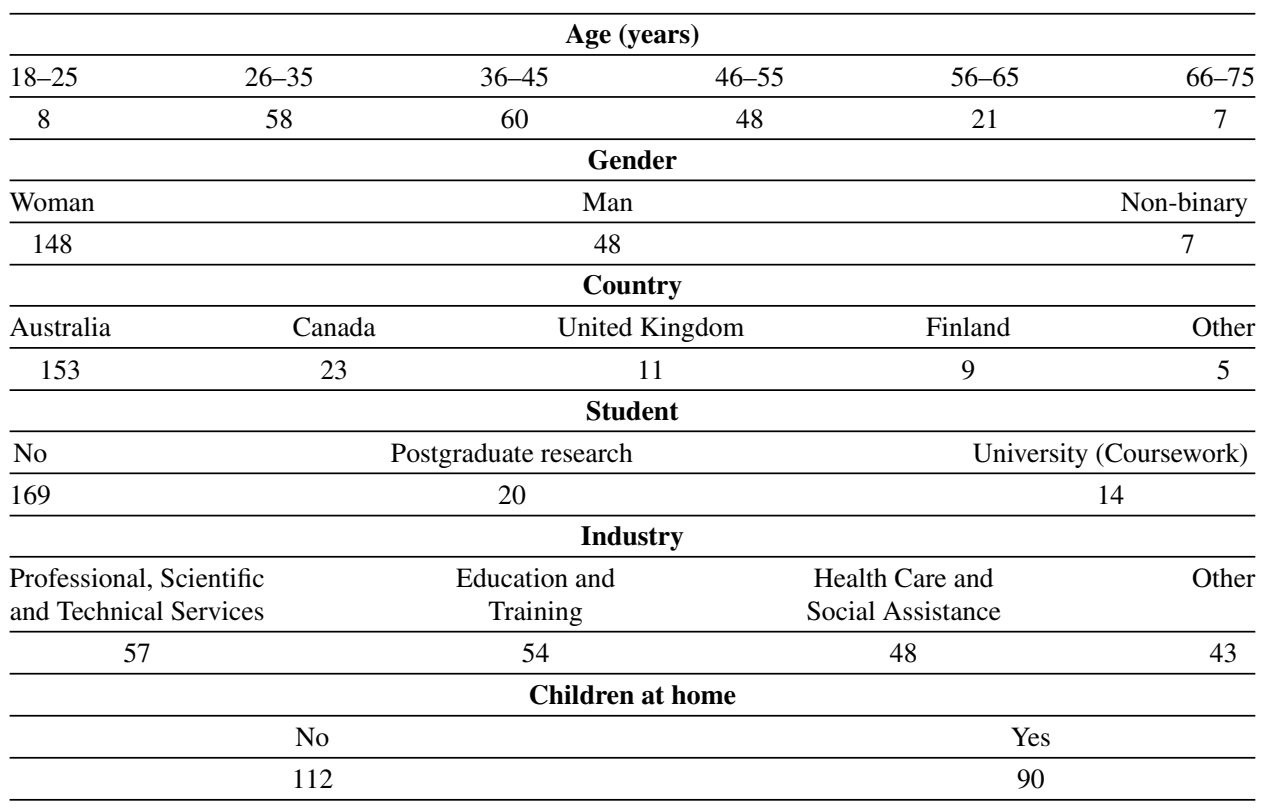

had the lowest response rate (median $75.5 \%$, IQR $62.1 \%-79.1 \%$ ). Of the remaining 55 questions, 51 had a response rate above $95 \%$. Table summaries of all non-free-text questions are supplied in the Supplementary Materials.

\subsection{Demographics}

As expected, given our sampling strategy, $75.7 \%$ $(n=152)$ of participants were Australian, $78.7 \%$ $(n=159)$ were working in Research, Healthcare or Education (tertiary) (Table 1). The ratio of female to male respondents was $3: 1$, with 7 non-binary participants. Less than half the participants had children.

\subsection{Experience with video-facilitated work practices pre-CRAZI and now (CRAZI)}

Prior to the CRAZI period, most participants worked from home occasionally, $4.0 \%(n=8)$ worked from home daily and $30 \%(n=33)$ of participants were entirely in the workplace (Fig. 1). All were using video-conferencing now.

Participants new to video-conferencing reported larger meetings with CRAZI. Meeting length was similar, most meetings lasting between 30-90 minutes (Fig. 1). While the average CRAZI meeting was less than 2 hours, $57.4 \%(n=118)$ of participants had experienced meetings of $>2$ hours, with some lasting up to 9 hours. Almost half the participants $(49.5 \%$, $n=94$ ) had experienced a CRAZI meeting with $>100$ participants; the largest meeting recorded was an internal COVID-19 meeting with 2000 participants. Another large notable meeting was a 700-participant meeting to learn how to use Zoom.

\subsection{Platforms and equipment supporting CRAZI}

CRAZI meeting platforms are shown in Fig. 2, most participants worked across multiple platforms. Headphone use was evenly split and internet problems were infrequent.

\subsection{Home location for CRAZI}

Around half the participants $(54.9 \%, n=112)$ had a dedicated home office. The remainder used bedrooms, living rooms, kitchens and outside the home (e.g. garden) for CRAZI. 50\% $(n=56)$ of those with a home office still moved around during CRAZI. Notable CRAZI locations included the laundry, kids' playroom, the toilet (more on that later), the car, or while using exercise equipment.

\subsection{CRAZI tolerance and adverse effects}

The median (IQR) number of tolerable hours of video-conferencing was 4 (IQR 2-5) per day; 
(a) Videoconferencing Present or Used

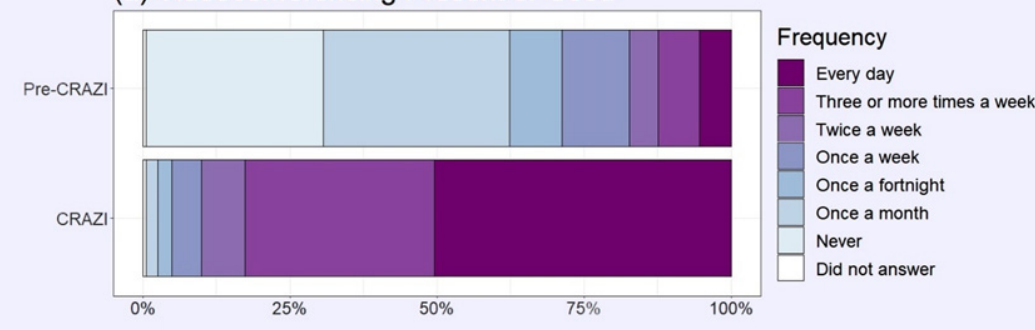

(b) Average Meeting Length

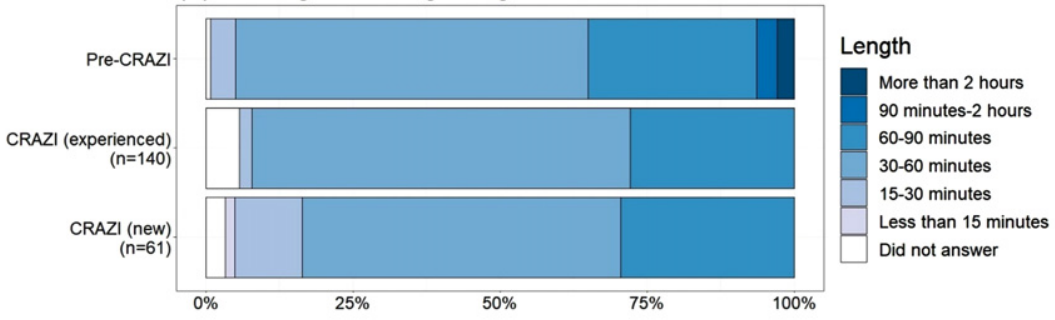

(c) Average Meeting Size

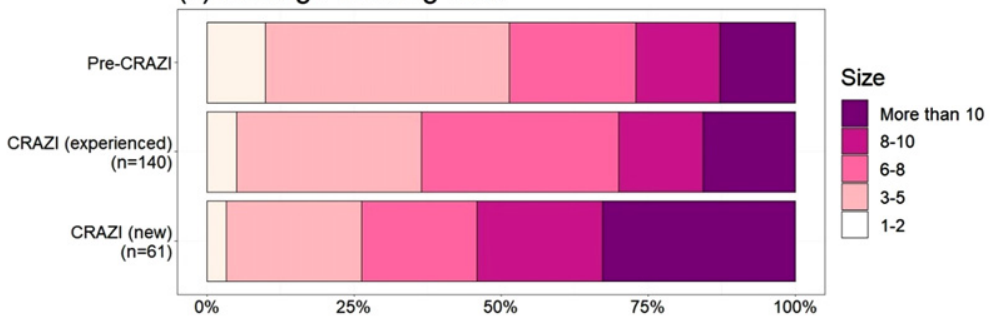

Fig. 1. Video-conferencing frequency, length and meeting size pre-CRAZI (face-to-face or virtual), and now (CRAZI), exploring prior experience with video-conferencing; experienced versus new.

$69 \%(n=139)$ listed fatigue as the main tolerance threshold reason (Fig. 3). Some participants felt CRAZI interfered with other work (e.g., "I still have non-CRAZI work to do"), and caring responsibilities contributed to maximum tolerable CRAZI hours.

\subsection{Managing CRAZI}

"Can you hear me now?" and "You're on mute" were common phrases (Fig. 4). Over half (61.7\%, $n=124$ ) of participants reported sometimes, often or always turning off their video nominating the following reasons; 1) improving internet connection stability $(51.5 \%, n=104), 2)$ taking a bio break or making a coffee $(30.2 \%, n=61), 3)$ looking after the needs of children $(17.3 \%, n=35)$ or 4$)$ avoiding others knowing you are multi-tasking $(32.2 \%, n=65)$. Interestingly, $15.8 \%(n=32)$ of participants did not like to see themselves. Multi-tasking during CRAZI was common with only $7.9 \%(n=8)$ of participants stating they rarely or never multi-tasked.

\subsection{CRAZI life and fashion}

The most common fashion approach with CRAZI was casual wear $(71.1 \%, n=143)$, with nudity the least frequent $(2.0 \% n=4)$, but not unheard of, fashion choice (Fig. 5). Most participants did not use a virtual background during CRAZI $(62.4 \%, n=136)$. The most common reasons reported for using a background were 'to make people laugh' $(63.6 \% n=42)$, for social points 'the background was cool' $(47.0 \%$ $n=31$ ), or for privacy $(45.5 \% n=30)$. Respondents nomination of funniest backgrounds included; toilet rolls, a bar, the CEOs office (when they are not the CEO), as well as a range of narcissistic options such as; the person sitting next to themselves or bringing themselves a cup of tea.

Seeing animals during CRAZI was common, the median (IQR) frequency of viewing an animal is the last week was 2 (IQR 1-3.75). While dogs (74.8\% $n=151)$ and cats $(63.4 \% n=128)$ were the most frequently reported animals seen during CRAZI, 


\section{(a) CRAZI Platforms}

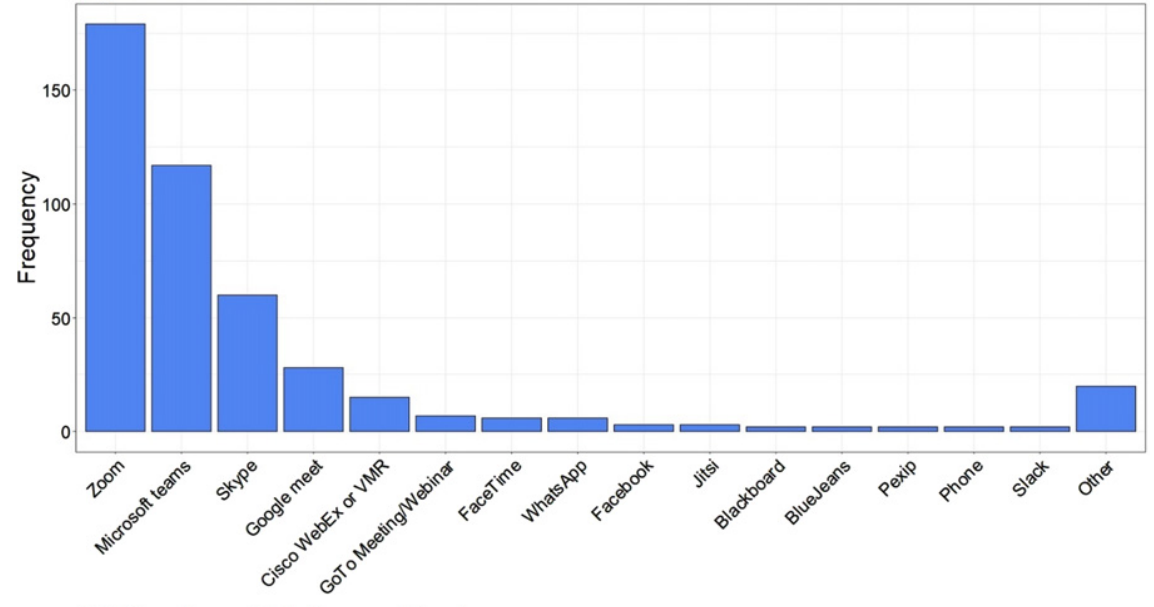

(b) Number of Platforms Used

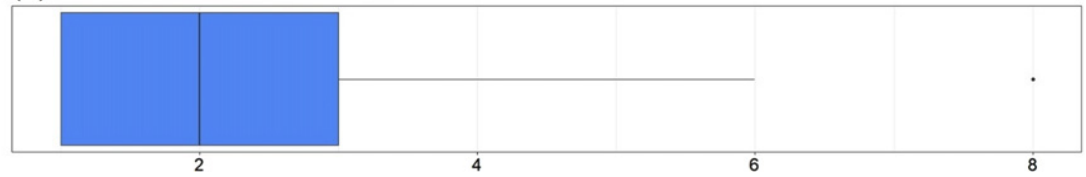

Fig. 2. Identified platform use for video-conferencing and work from home meetings (a) and median (IQR) number of platforms used per person (b).

(a) Easier or Harder?

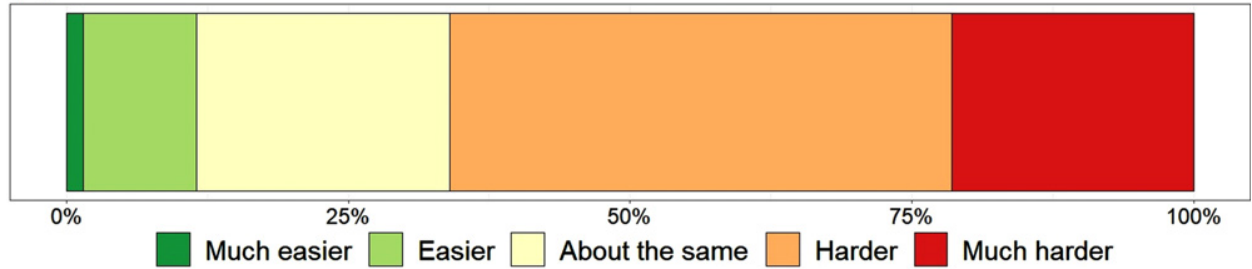

(b) More Tiring or Less Tiring?

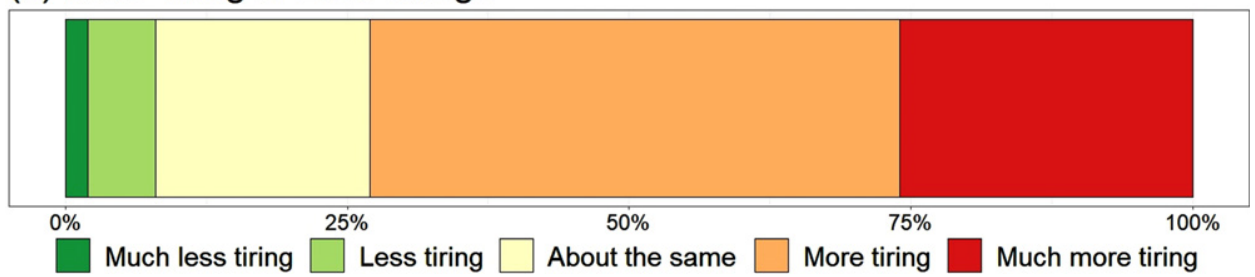

Fig. 3. Compared to pre-CRAZI, CRAZI video-conferencing was often rated as harder (a) and more tiring (b).

chickens, rabbits, goldfish, turtles, mice, rats, ponies and snakes were also reported.

\subsection{CRAZI behaviour}

Open field data exploring the experience of CRAZI are presented here.

\subsubsection{Differences in meeting behaviour pre-CRAZI to CRAZI}

Most respondents felt CRAZI meetings were different. Common positive themes included: 1) more attention to checking on peoples' wellbeing; 2) reduced formality of meetings and flattening of workplace hierarchy, potentially related to home location and more casual work attire; 3) integrating social 


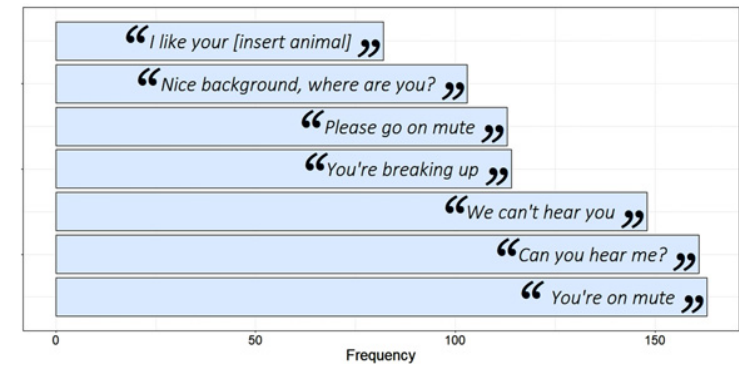

Fig. 4. "Can you hear me?" and "You're on mute" were the most common phrases heard during video-conferencing in the last week.

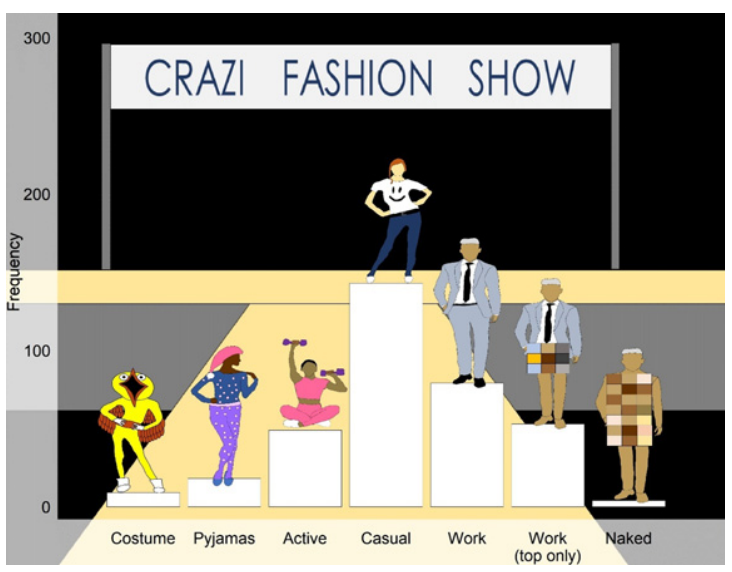

Fig. 5. Frequency of fashion choices for CRAZI meetings.

interactions into meetings to make up for loss of incidental workplace socialisation. Increasing experience and competence with video and other technologyassisted communication (e.g. Slack channels) were seen as better connecting people on other floors, in other buildings or interstate who would normally miss out on incidental exchanges. Increased accessibility and less travel time with video-based meetings was considered "really good news that ought to benefit everyone post-COVID" [Participant (P)188].

While some respondents reported increased length of meetings to accommodate social interactions, others reported shorter, more focused and transactional meetings. Common negative themes included: 1) "zoom fatigue" [P43]; crankiness "I feel like I am on zoom meetings all day, every day" [P202]; increased self-consciousness (because you can see yourself) and awkwardness with video-based interactions including feeling "disconnected only communicating with others through a screen" [P61]; "very sore throats if teaching all day" [P84] and 2) less enjoyment of work socialisation and less connection with others, "a strange rigour around causal conversations" [P200] and feeling obliged to interact, "it is slightly harder to excuse yourself from a video chat than from a real life coffee break" [P139].

\subsubsection{CRAZI etiquette}

Positively-viewed CRAZI etiquette included: 1) turn taking (not speaking over others, careful listening); 2) use of chat functions or raising hand to aid turn taking; 3) muting microphone when not talking; 4) patience and tolerance of technical challenges and helping others problem solve these challenges; 5) good facilitation so everyone understands standards, has input and the meeting is kept to time; 6) being attentive (not dual tasking) and asking after each other.

\subsubsection{CRAZI inappropriate behaviour}

Unsurprisingly, noted inappropriate CRAZI behaviour was often the opposite, i.e., talking over or interrupting others in the meeting, not being on mute when eating, slurping coffee, blowing noses, going to the toilet, typing etc. Leaving the meeting without notice, or obviously multi-tasking during a meeting. Several serious examples noted deliberate 'sabotage' of meetings, through incessant interruption, yelling, spamming the chat function, and three examples of unacceptable behaviour such as sexual references, sharing screens with inappropriate content and showing and mocking images of peers. Most respondents had not witnessed inappropriate behaviour. However, taking a CRAZI meeting in bed, or seeing others in a household wearing inappropriate or no clothing (!), screaming at children and taking (unmuted) phone calls and discussing patient details, or your peers, were other examples of inappropriate behaviour. Despite these, $59 \%(n=118)$ of participants did not believe a video-conferencing code of conduct was warranted. The desire for a code of conduct was higher when there was no code of conduct and lower when there was (Fig. 6).

\subsubsection{CRAZI behaviour-Confessions}

Some embarrassing behaviour was admitted by $55 \%(n=111)$ of respondents. The most common admissions were bathroom breaks, making coffee or getting a drink (alcohol), manicuring nails, eating a meal (generally breakfast), only dressing the top half to look professional and not wearing pants $(n=2$, see also Fig. 5). Only $16 \%(n=32)$ of participants professed innocence of any embarrassing behaviour. 


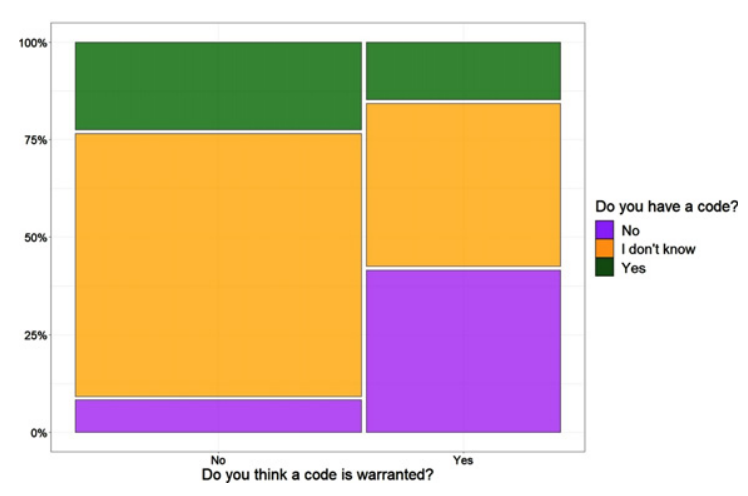

Fig. 6. Is a code of video-conferencing behaviour needed? Inverse relationship between having a code and wanting one, with a lot of fence sitting (I don't know).

\subsubsection{CRAZI - Funniest}

Pets and children dominated funniest CRAZI meeting responses. Children were generally considered to provide charming interruptions; for example, one child was seen delivering one strawberry at a time to his parent during a serious budget call. Animals also provided CRAZI meeting entertainment. A male CFO taking a meeting in his daughters' room with fairy wall motifs and a senior staff member dressed as a pickle were notable high points.

\subsubsection{Differences in CRAZI social interactions}

Many respondents reported marked differences between pre-COVID socialising and CRAZI socialising. CRAZI social was described as 'awkward', 'more formal', 'stilted' and 'less authentic' and 'less enjoyable'. Many respondents considered CRAZI socialising, particularly in larger groups to be harder, leading to fatigue. The challenge of broadening conversational topic away from COVID and people's stress and mental health was common. Opinions about whether CRAZI socialising was more inclusive was divided. While some participants felt that introverts have greater challenges socialising with CRAZI, some who identified as introverted or with mental health issues found the format easier to control and preferred CRAZI socialising. Improved accessibility due to distance, travel and less concern about dress standards were positively viewed. Finally, the home setting of most CRAZI socialisation was viewed as fostering more personal conversations with colleagues.

\subsubsection{CRAZI childcare}

Under half $(44.6 \%, n=89)$ the participants reported they had children at home. Of these $65.2 \%$ $(n=58)$ were home schooling. When asked to estimate the proportion or caring/schooling individuals were responsible for, men reported a median $50 \%$ (IQR 30\%-60\%) and women a median 60\% (IQR $50 \%-90 \%)$ share of responsibility. $76.6 \%(n=153)$ of all participants reported observing a child interrupting CRAZI one or more times in the last week, with the gender split for the person interrupted $66.2 \%$ $(n=131)$ women; $31.3 \%(n=62)$ men.

\subsection{The future of CRAZI}

While participants generally expressed positive views of video-conferencing and working from home, an even split was seen on preference to continue to work from home or return to previous arrangements after the pandemic (Fig. 7).

\section{Discussion}

The exponential uptake of virtual tools, with business apps like Zoom downloaded 62 million times in just a week during March in 2020 [11], is unprecedented. While video-conferencing has served us well, understanding patterns of use and behaviour can help refine tools and mitigate any potential harms. Optimising good CRAZI behaviour is paramount and this study contributes research to a growing body of anecdotal evidence about CRAZI.

Our relatively small sample was a limitation which prevented interrogation of CRAZI behaviour in different participant groups, e.g. in Boomers versus Zoomers, parents versus childfree participants, blankers (no video) and exhibitionists (video background (ab)users) etc [12]. Such exploration in larger samples may help us understand factors influencing CRAZI, or working from home preferences post pandemic. We acknowledge the complex intersections between self-reported fatigue, burnout and mental illness emerging in this global pandemic, and we did not explore these sensitive issues, which are important areas for further study.

\subsection{Zoom fatigue and the selfie complex}

As neuroscientists, the high reporting of 'Zoom fatigue', a form of mental exhaustion associated with video-conferencing, was interesting. This term is now common parlance. We believe it is not simply screen hours (median CRAZI tolerance 4 hours) that contribute to this phenomenon. Several hypotheses have 
(a) How do you feel about working from home now?

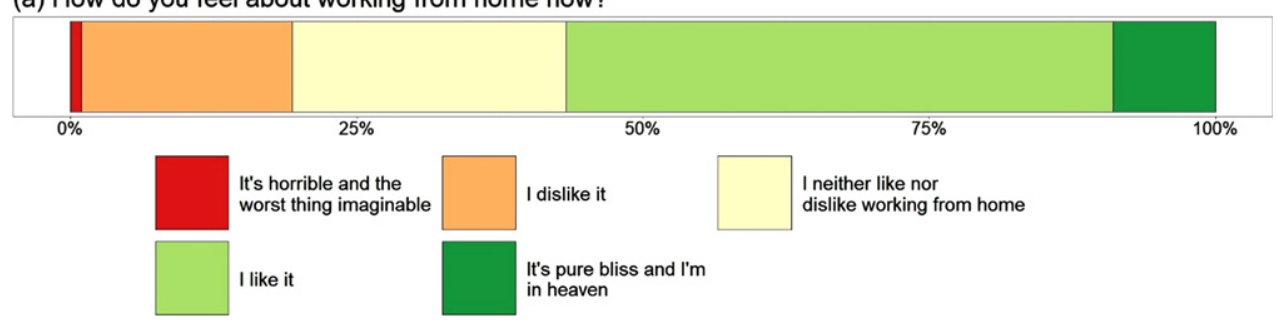

(b) Would you prefer to continue working from home or to return to previous work practices?

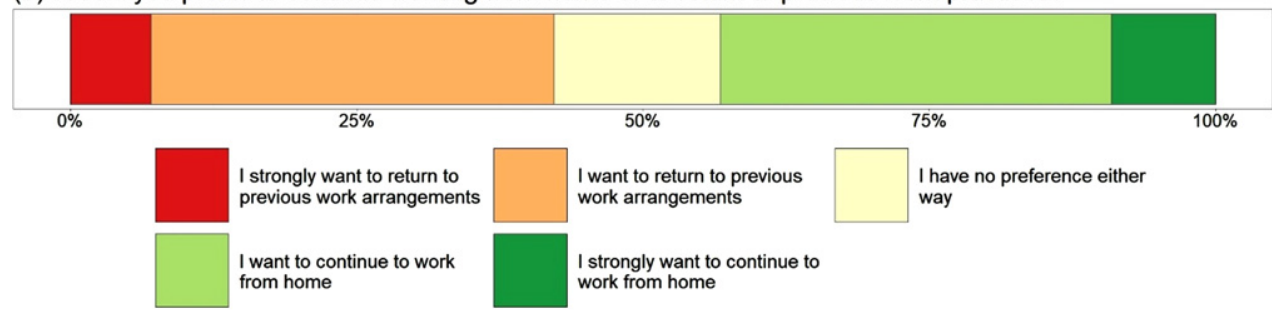

(c) Once the pandemic is over, I...

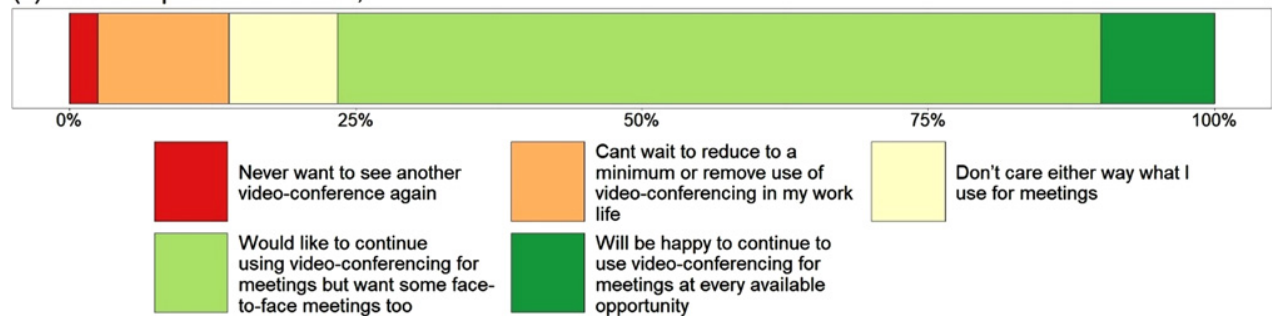

Fig. 7. View of working from home now (a), in the near future (b) and once the pandemic is over (c) showing strong support for ongoing use of video-conferencing, but evenly split preference for working from home.

been proposed. Is seeing your own face stressful? Viewing ourselves, versus others, activates different brain patterns and may increase cognitive load during CRAZI [13, 14]. If we see ourselves with negative expression, we may feel more intense emotions [15]. Even when our video is off, discerning important communication cues from others via a small rectangular screen, sometimes frozen in time, can contribute to cognitive load and fatigue. CRAZI makes it hard to detect non-verbal cues, so we must exert more effort with direct language, or over-the-top body language. Increased vigilance can deplete resources [16]. More experienced CRAZI users often multitasked, a behaviour that negatively impacts speech perception [17]. Limiting the degree of multi-tasking was recently recommended as one of a range of mitigation strategies [18]. Our data suggests that CRAZI social interaction does not elicit the same reward as face-to-face. Virtual social support can be as effective as face-to-face for stress buffering, however it must be provided by another human - a black screen is seemingly insufficient [19]. Noting that neuroatypical individuals may find CRAZI easier than in person interactions raises interesting considerations for inclusion moving forward.

\subsection{CRAZI children challenges}

While children were generally considered a charming interruption by observers, those responsible for caring for children begged to differ. Two academic parents juggling work with home schooling recently collected data on daily interruptions to their work by their children and reported an average of 15 times per hour, twice the rate experienced by emergency department doctors and nurses [20]. The impact of interruptions on vigilance is well documented [21] and as women are taking up the bulk of caring due to school closures [22], reductions in productivity will be disproportionately experienced. The burden and stress people experience may be mitigated using different strategies [18]. 


\section{Limitations}

The relatively small sample size recruited in this survey limited both the level of statistical analysis and interpretation of results which was justifiable. As our sample was underpowered for formal statistical testing, we opted not to perform formal hypothesis testing, as such an analysis would have uncontrolled type-I error inflation. For the same reason, we did not explore how factors such as gender, age, and prior experience working from home influenced a person's response to CRAZI.

The vast majority of respondents were from Australia, which is likely to be a result of our recruitment strategy, using the professional networks and social media of the authors of this paper. There are differences between countries in how much people spend working remotely [2] and the experiences people had with using online platforms before the pandemic in Australia might be different to people in other countries.

We did not explore the possible physical consequences of altered, and potentially inferior, home work stations on physical well-being in our survey. Recent work suggests a rising incidence of problems [23] which also may require mitigation strategies [24].

The over-representation of women respondents should be considered when interpreting these results. In a recent survey about work-family strategies during COVID-19, $36.6 \%$ of the dual incomes couples with young children surveyed employed "traditional" strategies to cope, where the female in the couple was primarily responsible for childcare [25]. Unfortunately, due to our small sample size, we were unable to explore gender differences further.

\section{Conclusions}

With some Australian companies announcing they will no longer require employees to return to the office for work after the pandemic ends [26], a new work culture is emerging. Our study found an appetite for more video-conferencing post pandemic, with positive views about continuing to work from home. This 'new normal' reflects an enormous paradigm shift. The blurring of lines between home and work has created a new, more relaxed work code. Getting to know peoples' pets was universally viewed as positive. The idea that pets in the workplace can reduce stress and improve personal and organisation well-being is not new [27]. Implementing it in the workplace is however complex. Working from home, with your pet, may offer the best of both worlds and, given that snakes and chickens are probably less acceptable visitors to the workplace than cats or dogs, is also more inclusive. The breadth of possible future research related to video-based interactions and working from home is enormous.

\section{Acknowledgments}

The authors wish to thank the participants who completed this survey amidst the early phase of the COVID-19 pandemic. The Florey Institute of Neuroscience and Mental Health acknowledges the infrastructure support of the Victorian State Government. JB was supported by NHMRC fellowship (ID1154904).

\section{Conflict of interest}

The authors declare no conflicts of interest related to this work.

\section{Supplementary data}

The supplementary files are available from https:// dx.doi.org/10.3233/WOR-210279.

\section{References}

[1] TUC. Homeworking up more than a quarter in last decade, TUC analysis shows 2019 [07 June 2021]. Available from: https://www.tuc.org.uk/news/homeworking-more-quarterlast-decade-tuc-analysis-shows.

[2] Messenger J, Vargas Llave O, Gschwind L, Boehmer S, Vermeylen G, Wilkens M. Eurofound Working Anytime, Anywhere: The Effects on the World of Work. Luxembourg: Publication Office of the European Union; 2017.

[3] Bureau of Labor Statistics (2016), American Time Use Survey - 2019 Results 2020 [07 June 2021]. Available from: www.bls.gov/news.release/pdf/atus.pdf

[4] Messenger J, Gschwind L. Three Generations of Telework: New ICT and the (R) Evolution From Home Office to Virtual Office. New Technology, Work and Employment. 2016;31(3):195-208.

[5] The Flex study: Global findings on the future of flexible work. Fuze; [07 June 2021]. Available from: https://www. fuze.com/future-of-work.

[6] Felstead A, Henseke G. Assessing the growth of remote working and its consequences for effort, well-being and work-life balance. New Technology, Work and Employmentpublished. 2017;32(3). 
[7] Hunter P. Remote working in research: An increasing usage of flexible work arrangements can improve productivity and creativity. EMBO Rep. 2019;20(1).

[8] Flores M. Understanding the challenges of remote working and it's impact to workers. International Journal of Business Marketing and Management. 2019;4(11):40-4.

[9] Grant C, Wallace L, Spurgeon P. An exploration of the psychological factors affecting remote e-worker's job effectiveness, well-being and work-life balance. Employee Relations. 2013;35(5):527-46.

[10] Team R. Language and Environment for Statistical Computing (3.4. 4). R Foundation for Statistical Computing. 2020.

[11] L. S. Video Conferencing Apps Surge from Coronavirus Impact: App Annie 2020 [cited 202030 March 2020]. Available from: https://www.appannie.com/en/insights/marketdata/video-conferencing-apps-surge-coronavirus/2020.

[12] Reid P. Different type of Zoomers 2020 [cited 2020 March 2020]. Available from: https://youtu.be/0fuTzJQ8Jls.

[13] Uddin LQ, Iacoboni M, Lange C, Keenan JP. The self and social cognition: the role of cortical midline structures and mirror neurons. Trends Cogn Sci. 2007;11(4):153-7.

[14] Uddin LQ, Kaplan JT, Molnar-Szakacs I, Zaidel E, Iacoboni M. Self-face recognition activates a frontoparietal "mirror" network in the right hemisphere: an event-related fMRI study. Neuroimage. 2005;25(3):926-35.

[15] Vergallito A, Mattavelli G, Gerfo EL, Anzani S, Rovagnati V, Speciale M, et al. Explicit and implicit responses of seeing own vs. others' emotions: an electromyographic study on the neurophysiological and cognitive basis of the selfmirroring technique. Front Psychol. 2020;11:433.

[16] Greenlee ET, DeLucia PR, Newton DC. Driver vigilance in automated vehicles: effects of demands on hazard detection performance. Hum Factors. 2019;61(3):474-87.

[17] Chiu F, Rakusen LL, Mattys SL. Cognitive load elevates discrimination thresholds of duration, intensity, and f0 for a synthesized vowel. J Acoust Soc Am. 2019;146(2):1077.
[18] Lopez-Leon S, Forero DA, Ruiz-Diaz P. Recommendations for working from home during the COVID-19 pandemic (and beyond). Work. 2020;66(2):371-5.

[19] Kothgassner OD, Goreis A, Kafka JX, Kaufmann M, Atteneder K, Beutl L, et al. Virtual social support buffers stress response: An experimental comparison of real-life and virtual support prior to a social stressor. J Behav Ther Exp Psychiatry. 2019;63:57-65.

[20] Weigl M, Beck J, Wehler M, Schneider A. Workflow interruptions and stress atwork: a mixed-methods study among physicians and nurses of a multidisciplinary emergency department. BMJ Open. 2017;7(12):e019074.

[21] Jorm CM, O'Sullivan G. Laptops and smartphones in the operating theatre - how does our knowledge of vigilance, multi-tasking and anaesthetist performance help us in our approach to this new distraction? Anaesth Intensive Care. 2012;40(1):71-8.

[22] Burki T. The indirect impact of COVID-19 on women. Lancet Infect Dis. 2020;20(8):904-5.

[23] Memari A, Shariat A, Anastasio AT. Rising incidence of musculoskeletal discomfort in the wake of the COVID-19 crisis. Work. 2020;66(4):751-3.

[24] Shariat A, Ghannadi S, Anastasio AT, Rostad M, Cleland JA. Novel stretching and strength-building exercise recommendations for computer-based workers during the COVID-19 quarantine. Work. 2020;66(4):739-49.

[25] Shockley KM, Clark MA, Dodd H, King EB. Work-family strategies during COVID-19: Examining gender dynamics among dual-earner couples with young children. J Appl Psychol. 2021;106(1):15-28.

[26] J. N. Atlassian tells employees they can work from home forever: CNBC 2020 [10 August 2020]. Available from: https://cnb.cx/2PyeY0q.

[27] Wells M, Perrine R. Critters in the cube farm: perceived psychological and organizational effects of pets in the workplace. J Occup Health Psychol. 2001;6(1):81-7. 